\title{
A Splitting Positive Definite Mixed Element Method for Second-Order Hyperbolic Equations
}

\author{
Jiansong Zhang, ${ }^{1}$ Danping Yang ${ }^{2}$ \\ ${ }^{1}$ School of Mathematics and System Sciences, Shandong University, Jinan, \\ Shandong 250100, China \\ ${ }^{2}$ Department of Mathematics, East China Normal University, Shanghai 200062, China
}

Received 2 November 2007; accepted 4 March 2008

Published online in Wiley InterScience (www.interscience.wiley.com).

DOI 10.1002/num.20363

In this article, we establish a new mixed finite element procedure, in which the mixed element system is symmetric positive definite, to solve the second-order hyperbolic equations. The convergence of the mixed element methods with continuous- and discrete-time scheme is proved. And the corresponding error estimates are given. Finally some numerical results are presented. @ 2008 Wiley Periodicals, Inc. Numer Methods Partial Differential Eq 00: 000-000, 2008

Keywords: convergence analysis; hyperbolic equation; mixed finite element; splitting positive definite system

\section{INTRODUCTION}

Hyperbolic equations describe the wave phenomena in the nature. They are very significant for many practical problems, e.g., hydrodynamics, displacement problems in porous media and vibrations of a membrane, acoustic vibrations of a gas, electromagnetic processes in nonconducting media.

Various numerical methods have been established for solving second-order hyperbolic problems. Continuous or discrete time Galerkin finite element methods are analyzed for linear or nonlinear second-order hyperbolic equations in several spaces, see [1-4]. The classical mixed element methods for the hyperbolic equations have been studied in [5-7]. However, the technique of the classical mixed method leads to some saddle point problems whose numerical solutions have been quite difficult because of losing positive definite properties.

The purpose of this article is, using the splitting technique as in [8], to formulate a new mixed element procedure to solve the second-order hyperbolic equation, in which the coefficient matrix of the mixed element system is symmetric positive definite. 
To illustrate our method, we consider our model as the following second-order hyperbolic problem:

$$
\begin{cases}c(x, t) \frac{\partial^{2} u}{\partial t^{2}}(x, t)-\nabla \cdot(\mathcal{A}(x, t) \nabla u(x, t))=f(x, t) & (x, t) \in \Omega \times(0, T] \\ u(x, t)=0, & x \in \partial \Omega, t>0 .\end{cases}
$$

with initial conditions

$$
\begin{cases}u(x, 0)=u_{0}(x), & x \in \Omega \\ u_{t}(x, 0)=q_{0}(x), & x \in \Omega\end{cases}
$$

where $\Omega$ is a bounded domain in $\mathbb{R}^{d}$ with boundary $\partial \Omega$. $c(x, t)>0$ and $\mathcal{A}=\left(a_{i j}\right)_{d \times d}$ the uniformly symmetric positive definite matrix function, i.e., there exists a constant $a_{0}>0$ such that

$$
a_{0} \sum_{i=1}^{d} \xi_{i}^{2} \leq \sum_{i, j=1}^{d} a_{i j}(x, t) \xi_{i} \xi_{j} \quad \forall \xi \in \mathbb{R}^{d}, \quad x \in \Omega
$$

The outline of this article is as follows. In Section II we give the continuous-time splitting positive definite finite element procedure for the model (1.1)-(1.2). Then we shall state and prove the convergence for this case. In Section III the discrete-time mixed element scheme will be defined and analyzed. In Section IV some numerical results are presented.

\section{CONTINUOUS-TIME ESTIMATES}

Throughout this article, usual definitions, notations, and norms of Sobolev spaces as in $[9,10]$ are used. $K$, with or without subscripts, denote generic positive constant, which may be different at their occurrences.

\section{A. Formulation of Splitting Positive Definite Mixed Element}

Introduce the function space $H(\operatorname{div} ; \Omega)=\left\{\omega \in\left[L^{2}(\Omega)\right]^{d} ; \operatorname{div} \omega \in L^{2}(\Omega)\right\}$ and the inner products in $L^{2}(\Omega)$ or $\left[L^{2}(\Omega)\right]^{d}$

$$
(u, v)=\int_{\Omega} u v d x, \forall u, v \in L^{2}(\Omega), \quad(\sigma, \omega)=\sum_{i=1}^{d} \int_{\Omega} \sigma_{i} \omega_{i} d x, \forall \sigma, \omega \in\left[L^{2}(\Omega)\right]^{d} .
$$

By introducing an unknown $\sigma=-\mathcal{A} \nabla u$. Let $\beta(x, t)=1 / c(x, t)$ and $\tilde{\mathcal{A}}=\mathcal{A}^{-1}$, a mixed weak form of (1.1) can be given by

$$
\left\{\begin{array}{lll}
\text { (a) } & \left(u_{t t}, v\right)+(\beta(x, t) \nabla \cdot \sigma, v)=(\beta(x, t) f, v) & \forall v \in L^{2}(\Omega), \\
\text { (b) } & (\tilde{\mathcal{A}} \sigma, \omega)-(u, \nabla \cdot \omega)=0 & \forall \omega \in H(\operatorname{div} ; \Omega) .
\end{array}\right.
$$

From (2.1b) we derive

$$
\left(\frac{\partial^{2}(\tilde{\mathcal{A}} \boldsymbol{\sigma})}{\partial t^{2}}, \omega\right)-\left(\frac{\partial^{2} u}{\partial t^{2}}, \nabla \cdot \omega\right)=0 \quad \forall \omega \in H(\operatorname{div} ; \Omega)
$$


By the definition of $\sigma$, we can define the following initial conditions:

$$
\left\{\begin{aligned}
(\tilde{\mathcal{A}} \sigma)(x, 0) & =-\nabla u_{0}(x) \\
\frac{\partial(\tilde{\mathcal{A}} \boldsymbol{\sigma})}{\partial t}(x, 0) & =-\nabla q_{0}(x)
\end{aligned}\right.
$$

Using the above initial conditions, we can easily show that the Eq. (2.2) is equivalent to the Eq. (2.1b).

Taking $v=\nabla \cdot \omega$ in (2.1a) for $\omega \in H(\operatorname{div} ; \Omega)$ and then substituting it into (2.2), we derive an equivalent mixed variational form of the system (2.1):

$$
\begin{cases}\text { (a) } \quad\left(\frac{\partial^{2}(\tilde{\mathcal{A}} \boldsymbol{\sigma})}{\partial t^{2}}, \omega\right)+(\beta \nabla \cdot \sigma, \nabla \cdot \omega)=(\beta f, \nabla \cdot \omega) & \forall \omega \in H(\operatorname{div} ; \Omega), \\ \text { (b) } \quad\left(\frac{\partial^{2} u}{\partial t^{2}}, v\right)+(\beta \nabla \cdot \sigma, v)=(\beta f, v) & \forall v \in L^{2}(\Omega) .\end{cases}
$$

Let $\mathcal{T}_{h_{u}}$ and $\mathcal{T}_{h_{\sigma}}$ be two families of quasi-regular partitions of the domain, which may be the same one or not, such that the elements in the partitions have the diameters bounded by $h_{u}$ and $h_{\sigma}$, respectively. Let $\mathcal{M}_{h_{u}} \subset L^{2}(\Omega)$ and $\mathcal{V}_{h_{\sigma}} \subset H$ (div; $\Omega$ ) be finite element spaces defined on the partitions $\mathcal{T}_{h_{u}}$ and $\mathcal{T}_{h_{\sigma}}$. Now we formulate a new mixed finite element procedure based on (2.3).

SPDME Scheme: Given an initial approximation $\left(u_{h}^{0}, \frac{\partial u_{h}}{\partial t}(x, 0), \sigma_{h}^{0}, \frac{\partial \sigma_{h}}{\partial t}(x, 0)\right) \in \mathcal{M}_{h_{u}} \times \mathcal{M}_{h_{u}} \times$ $\mathcal{V}_{h_{\sigma}} \times \mathcal{V}_{h_{\sigma}}$ such that

$$
\begin{array}{lll}
\text { (a) } & \left(u_{h}^{0}, v_{h}\right)=\left(u_{0}, v_{h}\right), & \forall v_{h} \in \mathcal{M}_{h_{u}}, \\
\text { (b) } \quad\left(\tilde{\mathcal{A}} \boldsymbol{\sigma}_{h}^{0}, \omega\right)=\left(u_{0}, \nabla \cdot \omega_{h}\right), & \forall \omega_{h} \in \mathcal{V}_{h_{\sigma}}, \\
\text { (c) } \quad\left(\frac{\left.\partial u_{h}(x, 0), v_{h}\right)=\left(q_{0}, v_{h}\right),}{\partial t},\right. & \forall v_{h} \in \mathcal{M}_{h_{u}}, \\
\text { (d) } & \left(\frac{\partial\left(\tilde{\mathcal{A}} \boldsymbol{\sigma}_{h}\right)}{\partial t}(x, 0), \omega\right)=\left(q_{0}, \nabla \cdot \omega_{h}\right), & \forall \omega_{h} \in \mathcal{V}_{h_{\sigma}} .
\end{array}
$$

Seek $\left(u_{h}, \sigma_{h}\right) \in \mathcal{M}_{h_{u}} \times \mathcal{V}_{h_{\sigma}}$ such that

$$
\begin{aligned}
& \text { (a) }\left(\frac{\partial^{2}\left(\tilde{\mathcal{A}} \sigma_{h}\right)}{\partial t^{2}}, \omega_{h}\right)+\left(\beta \nabla \cdot \sigma_{h}, \nabla \cdot \omega_{h}\right)=\left(\beta f, \nabla \cdot \omega_{h}\right) \text {, } \\
& \text { (b) } \quad\left(\frac{\partial^{2} u_{h}}{\partial t^{2}}, v_{h}\right) \quad \begin{aligned}
\forall \omega_{h} & \in \mathcal{V}_{h_{\sigma}}, \quad 0<t \leq T, \\
+ & +\left(\beta \nabla \cdot \sigma_{h}, v_{h}\right)=\left(\beta f, v_{h}\right),
\end{aligned} \\
& \forall v_{h} \in \mathcal{M}_{h_{u}}, \quad 0<t \leq T .
\end{aligned}
$$

It is easily seen that the Eq. (2.5a) is separated from the Eq. (2.5b) so that $\sigma_{h}$ can be solved independently from the Eq. (2.5a) and then $u_{h}$, if required, can be obtained from the Eq. (2.5b) almost explicitly.

Theorem 2.1. Assume that (1.3) holds. Then the system (2.5) with initial values defined by (2.4) has one unique solution.

Proof. Let $\left\{\omega_{h, i}\right\}_{i=1}^{N}$ and $\left\{v_{h, i}\right\}_{i=1}^{N}$ be basis functions of $\mathcal{V}_{h_{\sigma}}$ and $\mathcal{M}_{h_{u}}$, respectively, such that for each $\sigma_{h}(x, t) \in \mathcal{V}_{h_{\sigma}}$ and $u_{h}(x, t) \in \mathcal{M}_{h_{u}}$ there exist linear expressions $\sigma_{h}(x, t)=\sum_{i=1}^{N} a_{i}(t) \omega_{h, i}$ 
and $u_{h}(x, t)=\sum_{i=1}^{N} b_{i}(t) v_{h, i}$. Define vector-valued function $\boldsymbol{a}(t)=\left(a_{1}(t), \ldots, a_{N}(t)\right)^{\top}$, $\boldsymbol{b}(t)=\left(b_{1}(t), \ldots, b_{N}(t)\right)^{\top}, \boldsymbol{f}_{1}=\left(\left(\beta f, \nabla \cdot \omega_{h, 1}\right), \ldots,\left(\beta f, \nabla \cdot \omega_{h, N}\right)\right)^{\top}, \boldsymbol{f}_{2}(\boldsymbol{a})=((\beta(f-$ $\left.\left.\left.\nabla \cdot \sigma_{h}\right), v_{h, 1}\right), \ldots,\left(\beta\left(f-\nabla \cdot \sigma_{h}\right), v_{h, N}\right)\right)^{\top}$, and matrix functions

$$
B=\left(\tilde{\mathcal{A}}(i) \omega_{h, i}, \omega_{h, j}\right)_{N \times N}, \quad C=\left(\beta \nabla \cdot \omega_{h, i}, \nabla \cdot \omega_{h, j}\right)_{N \times N}, \quad D=\left(v_{h, i}, v_{h, j}\right)_{N \times N}
$$

where $\tilde{\mathcal{A}}(i)$ is the $i$ th row-vector of the matrix $\tilde{\mathcal{A}}$.

The system (2.5) may be rewritten as an equivalent matrix form:

$$
\begin{array}{ll}
\text { (a) } & \frac{d^{2}}{d t^{2}}(B \boldsymbol{a}(t))+C \boldsymbol{a}(t)=\boldsymbol{f}_{1}, \\
\text { (b) } & D \frac{d^{2} \boldsymbol{b}}{d t^{2}}=\boldsymbol{f}_{2}(\boldsymbol{a}), \\
\text { (c) } & \boldsymbol{a}(0)=\boldsymbol{a}_{0}, \quad \frac{d \boldsymbol{a}}{d t}(0)=\boldsymbol{q}_{0}, \\
\text { (d) } & \boldsymbol{b}(0)=\boldsymbol{b}_{0}, \quad \frac{d \boldsymbol{b}}{d t}(0)=\boldsymbol{q}_{1}\left(\boldsymbol{q}_{0}\right) .
\end{array}
$$

It is clear that matrixes $B, C$, and $D$ are symmetric positive definite. Let $\tilde{\boldsymbol{a}}=B \boldsymbol{a}$. The system (2.6) is rewritten as

$$
\begin{array}{ll}
\text { (a) } & \frac{d^{2} \tilde{\boldsymbol{a}}}{d t^{2}}=-C B^{-1} \tilde{\boldsymbol{a}}+\boldsymbol{f}_{1}, \\
\text { (b) } \frac{d^{2} \boldsymbol{b}}{d t^{2}}=D^{-1} \boldsymbol{f}_{2}\left(B^{-1} \tilde{\boldsymbol{a}}\right), \\
\text { (c) } \bar{a}(0)=B \boldsymbol{a}_{0}, \quad \frac{d \tilde{\boldsymbol{a}}}{d t}(0)=B \boldsymbol{q}_{0}+\frac{d B}{d t} \boldsymbol{a}_{0}, \\
\text { (d) } \boldsymbol{b}(0)=\boldsymbol{b}_{0}, \quad \frac{d \boldsymbol{b}}{d t}(0)=\boldsymbol{q}_{1}\left(\boldsymbol{q}_{0}\right) .
\end{array}
$$

The system (2.7) is an initial value problem of a system linear second-order ordinary differential equations. By virtue of the theory of ordinary differential equations, the system (2.7) has a unique solution, and so the system (2.5) has one unique solution. The proof of Theorem 2.1 is complete.

It is clear that the matching relation (i.e., LBB-condition ) between the mixed element spaces $\mathcal{V}_{h_{\sigma}}$ and $\mathcal{M}_{h_{u}}$, which is required by the classical mixed element spaces defined in [11-15], now is not necessary. From the viewpoint of computation, one can chose the usual continuous finite element spaces as $\mathcal{V}_{h_{\sigma}}$.

In the following part of this section, we will analyze the convergence of the SPDME Scheme and give the error estimate under the assumption that $\mathcal{V}_{h_{\sigma}}$ is one of the classical mixed elements in [11-15].

\section{B. Convergence Analysis and Error Estimate}

We assume that finite element spaces $\mathcal{V}_{h_{\sigma}}$ and $\mathcal{M}_{h_{u}}$ have the inverse property (see [16]) and approximate properties (see [11-15]) that there exist some integers $r, r_{1}, k>0$, such that, for $1 \leq q \leq \infty$ and $\forall \omega \in H(\operatorname{div} ; \Omega) \cap\left[W^{r+1, q}(\Omega)\right]^{d}$,

$$
\begin{aligned}
& \inf _{\omega_{h} \in \mathcal{V}_{h_{\sigma}}}\left\|\omega-\omega_{h}\right\|_{L^{q}(\Omega)} \leq K h_{\sigma}^{r+1}\|\omega\|_{W^{r+1, q}(\Omega)} ; \\
& \inf _{\omega_{h} \in \mathcal{V}_{h_{\sigma}}}\left\|\nabla \cdot\left(\omega-\omega_{h}\right)\right\|_{L^{q}(\Omega)} \leq K h_{\sigma}^{r_{1}}\|\nabla \cdot \omega\|_{W^{r_{1}, q}(\Omega)} ; \\
& \inf _{v_{h} \in \mathcal{M}_{h_{u}}}\left\|v-v_{h}\right\|_{L^{q}(\Omega)} \leq K h_{u}^{k+1}\|v\|_{W^{k+1, q}(\Omega)} \quad \forall v \in L^{2}(\Omega) \cap W^{k+1, q}(\Omega),
\end{aligned}
$$


where $r_{1}=r$ in cases of BDFM elements and BDM elements, or $r_{1}=r+1$ in cases of Nedelec elements and RT elements.

To analyze the convergence of the approximate solution determined by the SPDME scheme, we introduce some operators. It is well known that, in any one of the classical mixed finite element spaces, there exists an operator $\Pi_{h}$ from $H(\operatorname{div} ; \Omega)$ onto $\mathcal{V}_{h_{\sigma}}$, see [11-16], such that, for $1 \leq q \leq \infty$

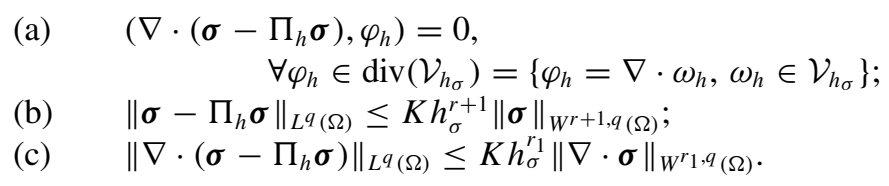

We also define the $L^{2}$-project operator $P_{M}$ from $L^{2}(\Omega)$ onto $\mathcal{M}_{h_{u}}$ such that
(a) $\quad\left(u-P_{M} u, v_{h}\right)=0 \quad \forall u \in L^{2}(\Omega), v_{h} \in \mathcal{M}_{h_{u}}$;
(b) $\quad\left\|u-P_{M} u\right\|_{L^{2}(\Omega)} \leq K h_{u}^{k+1}\|u\|_{H^{k+1}(\Omega)} \quad \forall u \in H^{k+1}(\Omega)$.

By use of the definitions of the operators $\Pi_{h}$ and $P_{M}$, we can easily obtain the following lemma.

Lemma 2.1. Suppose that the solution of system (2.3) has regular properties that $\partial^{2} u / \partial t^{2} \in$ $L^{2}\left(H^{k+1}(\Omega)\right), \partial^{2} \sigma / \partial t^{2} \in L^{2}\left(H^{r+1}(\Omega)\right), \partial u / \partial t \in L^{2}\left(H^{k+1}(\Omega)\right), \partial \sigma / \partial t \in L^{2}\left(H^{r+1}(\Omega)\right)$, then we have the following estimate

(a) $\left\|\nabla \cdot\left(\sigma-\Pi_{h} \boldsymbol{\sigma}\right)_{t}\right\|_{L^{q}(\Omega)} \leq K h_{\sigma}^{r_{1}}\left\|\nabla \cdot \sigma_{t}\right\|_{W^{r_{1}, q}(\Omega)}$,

(b) $\left\|\left(\sigma-\Pi_{h} \sigma\right)_{t}\right\|_{L^{q}(\Omega)} \leq K h_{\sigma}^{r+1}\left\|\sigma_{t}\right\|_{W^{r+1, q}(\Omega)}$,

(c) $\left\|\left(\sigma-\Pi_{h} \sigma\right)_{t t}\right\|_{L^{q}(\Omega)} \leq K h_{\sigma}^{r+1}\left\|\sigma_{t t}\right\|_{W^{r+1, q}(\Omega)}$,

(d) $\left\|\left(u-P_{M} u\right)_{t}\right\|_{L^{2}(\Omega)} \leq K h_{u}^{k+1}\left\|u_{t}\right\|_{H^{k+1}(\Omega)}$,

(e) $\left\|\left(u-P_{M} u\right)_{t t}\right\|_{L^{2}(\Omega)} \leq K h_{u}^{k+1}\left\|u_{t t}\right\|_{H^{k+1}(\Omega)}$.

Set $\theta=\sigma_{h}-\Pi_{h} \sigma, \rho=\sigma-\Pi_{h} \sigma, \xi=u_{h}-P_{M} u$, and $\eta=u-P_{M} u$. Subtracting (2.3) from (2.5), we get the residue equations:

(a) $\left(\frac{\partial^{2}(\tilde{\mathcal{A}} \theta)}{\partial t^{2}}, \omega_{h}\right)+\left(\beta \nabla \cdot \theta, \nabla \cdot \omega_{h}\right)$

$$
=\left(\frac{\partial^{2}(\tilde{\mathcal{A}} \rho)}{\partial t^{2}}, \omega_{h}\right)+\left(\beta \nabla \cdot \rho, \nabla \cdot \omega_{h}\right), \quad \forall \omega_{h} \in \mathcal{V}_{h_{\sigma}}, \quad 0<t \leq T,
$$

(b) $\left(\frac{\partial^{2} \xi}{\partial t^{2}}, v_{h}\right)+\left(\beta \nabla \cdot\left(\sigma_{h}-\sigma\right), v_{h}\right)=\left(\frac{\partial^{2} \eta}{\partial t^{2}}, v_{h}\right)$,

$$
\forall v_{h} \in \mathcal{M}_{h_{u}}, \quad 0<t \leq T .
$$


Theorem 2.2. Suppose that the finite element space $\mathcal{V}_{h_{\sigma}}$ is one of classical mixed elements in [11-15], that approximate properties (2.8) hold, that coefficients $c, \mathcal{A}$ in system (1.1) have the first- and second-order continuous derivatives on $t$, and the solution of system (2.3) has regular properties that $\partial^{2} u / \partial t^{2} \in L^{2}\left(H^{k+1}(\Omega)\right), \partial^{2} \sigma / \partial t^{2} \in L^{2}\left(H^{r+1}(\Omega)\right), \partial u / \partial t \in L^{2}\left(H^{k+1}(\Omega)\right)$, $\partial \boldsymbol{\sigma} / \partial t \in L^{2}\left(H^{r+1}(\Omega)\right), u \in L^{\infty}\left(H^{k+1}(\Omega)\right)$ and $\sigma \in L^{\infty}\left(H^{r+1}(\Omega)\right)$. Then we have the estimate

(a)

$$
\begin{aligned}
& \left\|\boldsymbol{\sigma}-\boldsymbol{\sigma}_{h}\right\|_{L^{\infty}\left(L^{2}(\Omega)\right)}+\left\|\frac{\partial}{\partial t}\left(\sigma-\sigma_{h}\right)\right\|_{L^{\infty}\left(L^{2}(\Omega)\right)} \\
& +\left\|\nabla \cdot\left(\sigma-\sigma_{h}\right)\right\|_{L^{\infty}\left(L^{2}(\Omega)\right)} \leq K h_{\sigma}^{r_{1}}
\end{aligned}
$$

(b)

$$
\left\|\frac{\partial}{\partial t}\left(u-u_{h}\right)\right\|_{L^{\infty}\left(L^{2}(\Omega)\right)} \leq K\left\{h_{\sigma}^{r_{1}}+h_{u}^{k+1}\right\}
$$

where $K$ is a constant independent of $h_{u}, h_{\sigma}$.

Proof. Take $\omega_{h}=\theta_{t}$ in (2.12a), we have

$$
\left(\frac{\partial^{2}(\tilde{\mathcal{A}} \theta)}{\partial t^{2}}, \theta_{t}\right)+\left(\beta \nabla \cdot \theta, \nabla \cdot \theta_{t}\right)=\left(\frac{\partial^{2}(\tilde{\mathcal{A}} \rho)}{\partial t^{2}}, \theta_{t}\right)+\left(\beta \nabla \cdot \rho, \nabla \cdot \theta_{t}\right)
$$

Note that

$$
\left(\frac{\partial^{2}(\tilde{\mathcal{A}} \theta)}{\partial t^{2}}, \theta_{t}\right)=\frac{1}{2} \frac{d}{d t}\left(\tilde{\mathcal{A}} \theta_{t}, \theta_{t}\right)+\frac{3}{2}\left(\tilde{\mathcal{A}}_{t} \theta_{t}, \theta_{t}\right)+\left(\tilde{\mathcal{A}}_{t t} \theta, \theta_{t}\right)
$$

and

$$
\left(\beta \nabla \cdot \theta, \nabla \cdot \theta_{t}\right)=\frac{1}{2} \frac{d}{d t}(\beta \nabla \cdot \theta, \nabla \cdot \theta)-\frac{1}{2}\left(\beta_{t} \nabla \cdot \theta, \nabla \cdot \theta\right) .
$$

Hence, we have

$$
\begin{aligned}
\int_{0}^{t} & {\left[\left(\frac{\partial^{2}(\tilde{\mathcal{A}} \theta)}{\partial \tau^{2}}, \theta_{\tau}\right)+\left(\beta \nabla \cdot \theta, \nabla \cdot \theta_{\tau}\right)\right] d \tau } \\
= & \frac{1}{2} \int_{0}^{t} \frac{d}{d \tau}\left[\left(\tilde{\mathcal{A}} \theta_{\tau}, \theta_{\tau}\right)+(\beta \nabla \cdot \theta, \nabla \cdot \theta)\right] d \tau+\frac{3}{2} \int_{0}^{t}\left(\tilde{\mathcal{A}}_{\tau} \theta_{\tau}, \theta_{\tau}\right) d \tau \\
& +\int_{0}^{t}\left(\tilde{\mathcal{A}}_{\tau \tau} \theta, \theta_{\tau}\right) d \tau-\frac{1}{2} \int_{0}^{t}(\beta \nabla \cdot \theta, \nabla \cdot \theta) d \tau \\
\geq \frac{1}{2}[ & \left.\left\|\tilde{\mathcal{A}}^{\frac{1}{2}} \theta_{t}(t)\right\|_{L^{2}(\Omega)}^{2}+\left\|\beta^{\frac{1}{2}} \nabla \cdot \theta(t)\right\|_{L^{2}(\Omega)}^{2}\right] \\
& -\frac{1}{2}\left[\left\|\tilde{\mathcal{A}}^{\frac{1}{2}} \theta_{t}(0)\right\|_{L^{2}(\Omega)}^{2}+\left\|\beta^{\frac{1}{2}} \nabla \cdot \theta(0)\right\|_{L^{2}(\Omega)}^{2}\right] \\
& -K \int_{0}^{t}\left[\left\|\theta_{\tau}\right\|_{L^{2}(\Omega)}^{2}+\|\theta\|_{L^{2}(\Omega)}^{2}+\|\nabla \cdot \theta\|_{L^{2}(\Omega)}^{2}\right] d \tau
\end{aligned}
$$

Numerical Methods for Partial Differential Equations DOI 10.1002/num 
Integrating (2.14) over $\tau$ in $(0, t]$, and using the above estimate, we can obtain

$$
\begin{aligned}
& \left\|\tilde{\mathcal{A}}^{\frac{1}{2}} \theta_{t}(t)\right\|_{L^{2}(\Omega)}^{2}+\left\|\beta^{\frac{1}{2}} \nabla \cdot \theta(t)\right\|_{L^{2}(\Omega)}^{2} \\
& \leq\left\|\tilde{\mathcal{A}}^{\frac{1}{2}} \theta_{t}(0)\right\|_{L^{2}(\Omega)}^{2}+\left\|\beta^{\frac{1}{2}} \nabla \cdot \theta(0)\right\|_{L^{2}(\Omega)}^{2}+2 \int_{0}^{t}\left(\beta \nabla \cdot \rho, \nabla \cdot \theta_{\tau}\right) d \tau \\
& \quad+K \int_{0}^{t}\left[\left\|\theta_{\tau}\right\|_{L^{2}(\Omega)}^{2}+\|\nabla \cdot \theta\|_{L^{2}(\Omega)}^{2}+\|\theta\|_{L^{2}(\Omega)}^{2}+\|\rho\|_{L^{2}(\Omega)}^{2}\right. \\
& \left.\quad+\left\|\rho_{\tau}\right\|_{L^{2}(\Omega)}^{2}+\left\|\rho_{\tau \tau}\right\|_{L^{2}(\Omega)}^{2}\right] d \tau .
\end{aligned}
$$

We know that

$$
\begin{aligned}
\int_{0}^{t} & \left(\beta \nabla \cdot \rho, \nabla \cdot \theta_{\tau}\right) d \tau \\
\quad & \int_{0}^{t} \frac{d}{d \tau}(\beta \nabla \cdot \rho, \nabla \cdot \theta) d \tau-\int_{0}^{t}\left(\beta_{\tau} \nabla \cdot \rho, \nabla \cdot \theta\right) d \tau-\int_{0}^{t}\left(\beta \nabla \cdot \rho_{\tau}, \nabla \cdot \theta\right) d \tau \\
\leq & (\beta(t) \nabla \cdot \rho(t), \nabla \cdot \theta(t))-(\beta(t) \nabla \cdot \rho(0), \nabla \cdot \theta(0)) \\
& \quad+K \int_{0}^{t}\left[\|\nabla \cdot \rho\|_{L^{2}(\Omega)}^{2}+\left\|\nabla \cdot \rho_{\tau}\right\|_{L^{2}(\Omega)}^{2}+\|\nabla \cdot \theta\|_{L^{2}(\Omega)}^{2}\right] d \tau
\end{aligned}
$$

Therefore, we can get

$$
\begin{aligned}
\left\|\theta_{t}(t)\right\|_{L^{2}(\Omega)}^{2}+\|\nabla \cdot \theta(t)\|_{L^{2}(\Omega)}^{2} \\
\leq K_{0}\left[\left\|\theta_{t}(0)\right\|_{L^{2}(\Omega)}^{2}+\|\nabla \cdot \theta(0)\|_{L^{2}(\Omega)}^{2}+\|\nabla \cdot \rho(t)\|_{L^{2}(\Omega)}^{2}+\|\nabla \cdot \rho(0)\|_{L^{2}(\Omega)}^{2}\right] \\
\quad+K \int_{0}^{t}\left[\left\|\theta_{\tau}\right\|_{L^{2}(\Omega)}^{2}+\|\nabla \cdot \theta\|_{L^{2}(\Omega)}^{2}+\|\theta\|_{L^{2}(\Omega)}^{2}+\|\rho\|_{L^{2}(\Omega)}^{2}\right. \\
\left.\quad+\|\nabla \cdot \rho\|_{L^{2}(\Omega)}^{2}+\left\|\rho_{\tau}\right\|_{L^{2}(\Omega)}^{2}+\left\|\nabla \cdot \rho_{\tau}\right\|_{L^{2}(\Omega)}^{2}+\left\|\rho_{\tau \tau}\right\|_{L^{2}(\Omega)}^{2}\right] d \tau \\
\quad \leq K\left\{h_{\sigma}^{2 r_{1}}+\int_{0}^{t}\left[\left\|\theta_{\tau}\right\|_{L^{2}(\Omega)}^{2}+\|\nabla \cdot \theta\|_{L^{2}(\Omega)}^{2}+\|\theta\|_{L^{2}(\Omega)}^{2} d \tau\right\} .\right.
\end{aligned}
$$

Adding $\|\theta\|_{L^{2}(\Omega)}^{2}$ to the above inequality and using the fact that $\theta(0)=0,\|\theta(t)\|_{L^{2}(\Omega)}^{2} \leq$ $T \int_{0}^{t}\left\|\theta_{\tau}\right\|_{L^{2}(\Omega)}^{2} d \tau$, we can obtain

$$
\begin{aligned}
& \|\theta(t)\|_{L^{2}(\Omega)}^{2}+\left\|\theta_{t}(t)\right\|_{L^{2}(\Omega)}^{2}+\|\nabla \cdot \theta(t)\|_{L^{2}(\Omega)}^{2} \\
& \quad \leq K\left\{h_{\sigma}^{2 r_{1}}+\int_{0}^{t}\left[\left\|\theta_{\tau}\right\|_{L^{2}(\Omega)}^{2}+\|\nabla \cdot \theta\|_{L^{2}(\Omega)}^{2}+\|\theta\|_{L^{2}(\Omega)}^{2} d \tau\right\} .\right.
\end{aligned}
$$

Using Gronwall's lemma, we can obtain

$$
\|\theta\|_{L^{\infty}\left(L^{2}(\Omega)\right)}+\left\|\theta_{t}\right\|_{L^{\infty}\left(L^{2}(\Omega)\right)}+\|\nabla \cdot \theta\|_{L^{\infty}\left(L^{2}(\Omega)\right)} \leq K h_{\sigma}^{r_{1}} .
$$

By use of (2.9) and Lemma 2.1, we get the estimate (2.13a).

Now we show that the estimate $(2.13 \mathrm{~b})$ holds. 
Taking $v_{h}=\xi$ in $(2.12 \mathrm{~b})$, we have

$$
\frac{1}{2} \frac{d}{d t}\left\|\xi_{t}\right\|_{L^{2}(\Omega)}^{2}=-\left(\beta \nabla \cdot\left(\sigma_{h}-\sigma\right), \xi_{t}\right)+\left(\frac{\partial^{2} \eta}{\partial t^{2}}, \xi_{t}\right) .
$$

Integrating (2.16) over $\tau$ in $(0, t]$, we can obtain

$$
\begin{aligned}
& \left\|\xi_{t}(t)\right\|_{L^{2}(\Omega)}^{2} \leq\left\|\xi_{t}(0)\right\|_{L^{2}(\Omega)}^{2} \\
& \quad+K \int_{0}^{t}\left[\left\|\nabla \cdot\left(\sigma_{h}-\sigma\right)\right\|_{L^{2}(\Omega)}^{2}+\left\|\eta_{\tau \tau}\right\|_{L^{2}(\Omega)}^{2}+\left\|\xi_{\tau}\right\|_{L^{2}(\Omega)}^{2}\right] d \tau .
\end{aligned}
$$

By use of Lemma 2.1, (2.13a) and Gronwall's lemma, we get

$$
\left\|\xi_{t}\right\|_{L^{\infty}\left(L^{2}(\Omega)\right)} \leq K\left\{h_{\sigma}^{r_{1}}+h_{u}^{k+1}\right\} .
$$

Using Lemma 2.1 again, we easily get the estimate (2.13b). This ends the proof of Theorem 2.2 .

\section{DISCRETE-TIME ESTIMATES}

\section{A. Formulation of Fully-discrete Scheme}

Let $J$ be a positive integer and let $\tau=T / J$ denote the time increment. For any function $r$ defined at the times $n \tau, n=0, \ldots, J$, denote by $r^{n}$ the function at $t=t^{n}=n \tau$; We shall use this notation for functions defined for all time as well as those defined only at $t=n \tau$. Some other notations we shall use are

$$
\begin{aligned}
& r^{n+\frac{1}{2}}=\left(r^{n+1}+r^{n}\right) / 2, \\
& r^{n, \theta}=\theta r^{n+1}+(1-2 \theta) r^{n}+\theta r^{n-1}, \\
& \partial_{t} r^{n+\frac{1}{2}}=\left(r^{n+1}-r^{n}\right) / \tau, \\
& \partial_{t}^{2} r^{n}=\left(r^{n+1}-2 r^{n}+r^{n-1}\right) / \tau^{2}, \\
& \delta_{t} r^{n}=\left(r^{n+1}-r^{n-1}\right) / 2 \tau .
\end{aligned}
$$

The Eq. (2.3) has the following equivalent formulation

$$
\left\{\begin{array}{c}
\left(\text { a) } \quad\left(\partial_{t}^{2}(\tilde{\mathcal{A}} \boldsymbol{\sigma})^{n}, \omega\right)+\left((\beta \nabla \cdot \boldsymbol{\sigma})^{n, \frac{1}{4}}, \nabla \cdot \omega\right)\right. \\
=\left((\beta f)^{n, \frac{1}{4}}, \nabla \cdot \omega\right)+\left(R_{1}^{n}, \omega\right) \quad \forall \omega \in H(\operatorname{div} ; \Omega), \\
\text { (b) } \quad\left(\partial_{t}^{2} u^{n}, v\right)+\left((\beta \nabla \cdot \sigma)^{n, \frac{1}{4}}, v\right)=\left((\beta f)^{n, \frac{1}{4}}+R_{2}^{n}, v\right) \quad \forall v \in L^{2}(\Omega),
\end{array}\right.
$$

where

$$
\begin{aligned}
& R_{1}^{n}=\left(\partial_{t}^{2}(\tilde{\mathcal{A}} \boldsymbol{\sigma})^{n}-\frac{\partial^{2}(\tilde{\mathcal{A}} \boldsymbol{\sigma})}{\partial t^{2}}\right)=O\left(\tau^{2} \frac{\partial^{4}(\tilde{\mathcal{A}} \boldsymbol{\sigma})}{\partial t^{4}}\right) \\
& R_{2}^{n}=\partial_{t}^{2} u^{n}-\frac{\partial^{2} u}{\partial t^{2}}=O\left(\tau^{2} \frac{\partial^{4} u}{\partial t^{4}}\right) .
\end{aligned}
$$


Now we formulate a fully-discrete splitting positive definite mixed element procedure based on (3.2).

Fully-discrete SPDME Scheme: Given initial value $\left(u_{h}^{0}, \sigma_{h}^{0}\right) \in \mathcal{M}_{h_{u}} \times \mathcal{V}_{h_{\sigma}}$ such that

$$
\begin{aligned}
& \text { (a) }\left(u_{h}^{0}, v_{h}\right)=\left(u_{0}, v_{h}\right), \quad \forall v_{h} \in \mathcal{M}_{h_{u}}, \\
& \text { (b) }\left(\tilde{\mathcal{A}}(x, 0) \sigma_{h}^{0}, \omega_{h}\right)=\left(u_{0}, \nabla \cdot \omega_{h}\right), \quad \forall \omega_{h} \in \mathcal{V}_{h_{\sigma}} .
\end{aligned}
$$

and $\left(u_{h}^{1}, \sigma_{h}^{1}\right) \in \mathcal{M}_{h_{u}} \times \mathcal{V}_{h_{\sigma}}$ such that

$$
\begin{aligned}
& \text { (a) }\left(\frac{u_{h}^{1}-u_{h}^{-1}}{2 \tau}, v_{h}\right)=\left(q_{0}, v_{h}\right), \quad \forall v_{h} \in \mathcal{M}_{h_{u}}, \\
& \text { (b) }\left(\frac{\tilde{\mathcal{A}}(x, \tau) \sigma_{h}^{1}-\tilde{\mathcal{A}}(x, 0) \sigma_{h}^{-1}}{2 \tau}, \omega_{h}\right)=\left(q_{0}, \nabla \cdot \omega_{h}\right), \quad \forall \omega_{h} \in \mathcal{V}_{h_{\sigma}} .
\end{aligned}
$$

For $n=1,2,3, \ldots, J-1$, seek $\left(u_{h}^{n+1}, \boldsymbol{\sigma}_{h}^{n+1}\right) \in \mathcal{M}_{h_{u}} \times \mathcal{V}_{h_{\sigma}}$ such that

$$
\begin{aligned}
& \text { (a) }\left(\tilde{\mathcal{A}}_{n} \partial_{t}^{2} \boldsymbol{\sigma}_{h}^{n}, \omega_{h}\right)+\left(\beta_{n} \nabla \cdot \boldsymbol{\sigma}_{h}^{n, \frac{1}{4}}, \nabla \cdot \omega_{h}\right)=\left((\beta f)^{n, \frac{1}{4}}, \nabla \cdot \omega_{h}\right), \quad \forall \omega_{h} \in \mathcal{V}_{h_{\sigma}}, \\
& \text { (b) }\left(\partial_{t}^{2} u_{h}^{n}, v_{h}\right)+\left(\beta_{n} \nabla \cdot \boldsymbol{\sigma}_{h}^{n, \frac{1}{4}}, v_{h}\right)=\left((\beta f)^{n, \frac{1}{4}}, v_{h}\right), \quad \forall v_{h} \in \mathcal{M}_{h_{u}} .
\end{aligned}
$$

It is easily seen that the following result holds.

Theorem 3.1. Assume that (1.3) holds. Then the system (3.5) with initial values defined by (3.4) and (3.4) has one unique solution.

\section{B. Convergence Analysis and Error Estimate}

Similar to Lemma 2.1, we have the following lemma.

Lemma 3.1. Suppose that the solution of system (2.3) has regular properties that $\partial^{2} u / \partial t^{2} \in$ $L^{2}\left(H^{k+1}(\Omega)\right), \partial^{2} \sigma / \partial t^{2} \in L^{2}\left(H^{r+1}(\Omega)\right), \partial u / \partial t \in L^{2}\left(H^{k+1}(\Omega)\right), \partial \sigma / \partial t \in L^{2}\left(H^{r+1}(\Omega)\right)$, then we have the following estimate

$$
\begin{aligned}
& \text { (a) }\left\|\partial_{t}\left(\boldsymbol{\sigma}-\Pi_{h} \boldsymbol{\sigma}\right)\right\|_{\tilde{L}^{\infty}\left(L^{q}(\Omega)\right)} \leq K h_{\sigma}^{r+1}, \\
& \text { (b) }\left\|\partial_{t}\left(u-P_{M} u\right)\right\|_{\tilde{L}^{\infty}\left(L^{q}(\Omega)\right)} \leq K h_{u}^{k+1},
\end{aligned}
$$

where for functions $\gamma$ with values at discrete times,

$$
\|\gamma\|_{\tilde{L}^{\infty}(\mathcal{X})}=\max _{0 \leq n<J}\left\|\gamma^{n+1 / 2}\right\|_{\mathcal{X}}
$$


Theorem 3.2. Suppose that $\partial^{4} u / \partial t^{4} \in L^{2}\left(L^{2}(\Omega)\right), \partial^{4} \sigma / \partial t^{4} \in L^{2}\left(\left[L^{2}(\Omega)\right]^{d}\right), \partial^{2} u / \partial t^{2} \in$ $L^{2}\left(H^{k+1}(\Omega)\right), \partial^{2} \sigma / \partial t^{2} \in L^{2}\left(H^{r+1}(\Omega)\right), u \in L^{\infty}\left(H^{k+1}(\Omega)\right)$, and $\sigma \in L^{\infty}\left(H^{r+1}(\Omega)\right)$. Let $\left(u_{h}, \sigma_{h}\right)$ denote the solution of the fully-discrete SPDME scheme given by (3.5). Then there exists a constant K such that

$$
\text { (a) } \begin{aligned}
& \left\|\partial_{t}\left(\sigma-\sigma_{h}\right)\right\|_{\tilde{L}^{\infty}\left(L^{2}(\Omega)\right)} \\
& +\left\|\nabla \cdot\left(\sigma-\sigma_{h}\right)\right\|_{\tilde{L}^{\infty}\left(L^{2}(\Omega)\right)} \leq K\left\{h_{\sigma}^{r_{1}}+\tau^{2}\right\}
\end{aligned}
$$

(b) $\left\|\partial_{t}\left(u-u_{h}\right)\right\|_{\tilde{L}^{\infty}\left(L^{2}(\Omega)\right)} \leq K\left\{h_{\sigma}^{r_{1}}+h_{u}^{k+1}+\tau^{2}\right\}$.

Proof. Set $\theta^{n}=\boldsymbol{\sigma}_{h}^{n}-\Pi_{h} \boldsymbol{\sigma}^{n}$ and $\rho^{n}=\boldsymbol{\sigma}_{h}^{n}-\Pi_{h} \boldsymbol{\sigma}^{n}$. Subtracting (3.2a) from (3.5a), we can easily obtain

$$
\begin{aligned}
\left(\tilde{\mathcal{A}}_{n} \partial_{t}^{2} \theta^{n}, \omega_{h}\right)+\left(\beta_{n} \nabla \cdot \theta^{n, \frac{1}{4}}, \nabla \cdot \omega_{h}\right) \\
=\left(\tilde{\mathcal{A}}_{n} \partial_{t}^{2} \rho^{n}, \omega_{h}\right)+\left(\beta_{n} \nabla \cdot \rho^{n, \frac{1}{4}}, \nabla \cdot \omega_{h}\right) \\
\quad+\left(\partial_{t}^{2}(\tilde{\mathcal{A}} \boldsymbol{\sigma})^{n}-\tilde{\mathcal{A}}_{n} \partial_{t}^{2} \boldsymbol{\sigma}^{n}, \omega_{h}\right)-\left(R_{1}^{n}, \omega_{h}\right) \\
\quad+\left(\beta_{n} \nabla \cdot \boldsymbol{\sigma}^{n, \frac{1}{4}}-(\beta \nabla \cdot \boldsymbol{\sigma})^{n, \frac{1}{4}}, \nabla \cdot \omega_{h}\right) .
\end{aligned}
$$

Taking $\omega_{h}=2 \delta_{t} \theta^{n}$ in (3.9), we can obtain

$$
\begin{aligned}
\left(\tilde{\mathcal{A}}_{n}\left(\partial_{t} \theta^{n+\frac{1}{2}}-\partial_{t} \theta^{n-\frac{1}{2}}\right), \partial_{t} \theta^{n+\frac{1}{2}}+\partial_{t} \theta^{n-\frac{1}{2}}\right) \\
\quad+\left(\beta_{n}\left(\nabla \cdot \theta^{n+\frac{1}{2}}+\nabla \cdot \theta^{n-\frac{1}{2}}\right), \nabla \cdot \theta^{n+\frac{1}{2}}-\nabla \cdot \theta^{n-\frac{1}{2}}\right) \\
=\tau\left(\tilde{\mathcal{A}}_{n} \partial_{t}^{2} \rho^{n}-R_{1}^{n}, \partial_{t} \theta^{n+\frac{1}{2}}+\partial_{t} \theta^{n-\frac{1}{2}}\right) \\
\quad+2\left(\beta_{n} \nabla \cdot \rho^{n, \frac{1}{4}}, \nabla \cdot \theta^{n+\frac{1}{2}}-\nabla \cdot \theta^{n-\frac{1}{2}}\right) \\
\quad+\tau\left(\partial_{t}^{2}(\tilde{\mathcal{A}} \boldsymbol{\sigma})^{n}-\tilde{\mathcal{A}}_{n} \partial_{t}^{2} \boldsymbol{\sigma}^{n}, \partial_{t} \theta^{n+\frac{1}{2}}+\partial_{t} \theta^{n-\frac{1}{2}}\right) \\
\quad+2\left(\beta_{n} \nabla \cdot \boldsymbol{\sigma}^{n, \frac{1}{4}}-(\beta \nabla \cdot \boldsymbol{\sigma})^{n, \frac{1}{4}}, \nabla \cdot \theta^{n+\frac{1}{2}}-\nabla \cdot \theta^{n-\frac{1}{2}}\right) .
\end{aligned}
$$

Note that

$$
\begin{aligned}
& \left(\tilde{\mathcal{A}}_{n}\left(\partial_{t} \theta^{n+\frac{1}{2}}-\partial_{t} \theta^{n-\frac{1}{2}}\right), \partial_{t} \theta^{n+\frac{1}{2}}+\partial_{t} \theta^{n-\frac{1}{2}}\right) \\
& \quad=\left\|\tilde{\mathcal{A}}_{n}^{\frac{1}{2}} \partial_{t} \theta^{n+\frac{1}{2}}\right\|_{L^{2}(\Omega)}^{2}-\left\|\tilde{\mathcal{A}}_{n-1}^{\frac{1}{2}} \partial_{t} \theta^{n-\frac{1}{2}}\right\|_{L^{2}(\Omega)}^{2}-\tau\left\|\partial_{t} \tilde{\mathcal{A}}_{n-\frac{1}{2}}^{\frac{1}{2}} \partial_{t} \theta^{n-\frac{1}{2}}\right\|_{L^{2}(\Omega)}^{2}
\end{aligned}
$$

and

$$
\begin{aligned}
& \left(\beta_{n}\left(\nabla \cdot \theta^{n+\frac{1}{2}}+\nabla \cdot \theta^{n-\frac{1}{2}}\right), \nabla \cdot \theta^{n+\frac{1}{2}}-\nabla \cdot \theta^{n-\frac{1}{2}}\right) \\
& \quad=\left\|\beta_{n}^{\frac{1}{2}} \nabla \cdot \theta^{n+\frac{1}{2}}\right\|_{L^{2}(\Omega)}^{2}-\left\|\beta_{n-1}^{\frac{1}{2}} \nabla \cdot \theta^{n-\frac{1}{2}}\right\|_{L^{2}(\Omega)}^{2}-\tau\left\|\partial_{t} \beta_{n-\frac{1}{2}}^{\frac{1}{2}} \nabla \cdot \theta^{n-\frac{1}{2}}\right\|_{L^{2}(\Omega)}^{2} .
\end{aligned}
$$


Substituting these equations into (3.10) and summing from 1 to $n$, we can obtain

$$
\begin{aligned}
& \left\|\tilde{\mathcal{A}}_{n}^{\frac{1}{2}} \partial_{t} \theta^{n+\frac{1}{2}}\right\|_{L^{2}(\Omega)}^{2}+\left\|\beta_{n}^{\frac{1}{2}} \nabla \cdot \theta^{n+\frac{1}{2}}\right\|_{L^{2}(\Omega)}^{2} \\
& =\left\|\tilde{\mathcal{A}}_{0}^{\frac{1}{2}} \partial_{t} \theta^{\frac{1}{2}}\right\|_{L^{2}(\Omega)}^{2}+\left\|\beta_{0}^{\frac{1}{2}} \nabla \cdot \theta^{\frac{1}{2}}\right\|_{L^{2}(\Omega)}^{2} \\
& \quad+\tau \sum_{k=1}^{n}\left[\left\|\partial_{t} \tilde{\mathcal{A}}_{k-\frac{1}{2}}^{\frac{1}{2}} \partial_{t} \theta^{k-\frac{1}{2}}\right\|_{L^{2}(\Omega)}^{2}+\left\|\partial_{t} \beta_{k-\frac{1}{2}}^{\frac{1}{2}} \nabla \cdot \theta^{k-\frac{1}{2}}\right\|_{L^{2}(\Omega)}^{2}\right] \\
& \quad+\tau \sum_{k=1}^{n}\left(\tilde{\mathcal{A}}_{k} \partial_{t}^{2} \rho^{k}-R_{1}^{k}, \partial_{t} \theta^{k+\frac{1}{2}}+\partial_{t} \theta^{k-\frac{1}{2}}\right) \\
& \quad+2 \sum_{k=1}^{n}\left(\beta_{k} \nabla \cdot \rho^{k, \frac{1}{4}}, \nabla \cdot \theta^{k+\frac{1}{2}}-\nabla \cdot \theta^{k-\frac{1}{2}}\right) \\
& \quad+\tau \sum_{k=1}^{n}\left(\partial_{t}^{2}(\tilde{\mathcal{A}} \boldsymbol{\sigma})^{k}-\tilde{\mathcal{A}}_{k} \partial_{t}^{2} \boldsymbol{\sigma}^{k}, \partial_{t} \theta^{k+\frac{1}{2}}+\partial_{t} \theta^{k-\frac{1}{2}}\right) \\
& \quad+2 \sum_{k=1}^{n}\left(\beta_{k} \nabla \cdot \boldsymbol{\sigma}^{k, \frac{1}{4}}-(\beta \nabla \cdot \boldsymbol{\sigma})^{k, \frac{1}{4}}, \nabla \cdot \theta^{k+\frac{1}{2}}-\nabla \cdot \theta^{k-\frac{1}{2}}\right) \\
& =\left\|\tilde{\mathcal{A}}_{0}^{\frac{1}{2}} \partial_{t} \theta^{\frac{1}{2}}\right\|_{L^{2}(\Omega)}^{2}+\left\|\beta_{0}^{\frac{1}{2}} \nabla \cdot \theta^{\frac{1}{2}}\right\|_{L^{2}(\Omega)}^{2}+\sum_{i=1}^{5} E_{i} .
\end{aligned}
$$

Now, we estimate the bounds of $E_{i}, i=1,2, \ldots, 5$.

Firstly, we estimate the bound of $E_{1}$. We can easily obtain

$$
E_{1} \leq K \tau \sum_{k=1}^{n}\left[\left\|\partial_{t} \theta^{k-\frac{1}{2}}\right\|_{L^{2}(\Omega)}^{2}+\left\|\nabla \cdot \theta^{k-\frac{1}{2}}\right\|_{L^{2}(\Omega)}^{2}\right]
$$

And then, using the inequality $\tau \sum_{k}\left\|R_{1}^{k}\right\|_{L^{2}(\Omega)}^{2} \leq K \tau^{4}\left\|\frac{\partial^{4}(\tilde{\mathcal{A}} \sigma)}{\partial t^{4}}\right\|_{L^{2}\left(L^{2}(\Omega)\right)}^{2}$ and (2.11c), we can derive

$$
\begin{aligned}
E_{2} & \leq K \tau \sum_{k=1}^{n}\left(\left\|\partial_{t}^{2} \rho^{k}\right\|_{L^{2}(\Omega)}+\left\|R_{1}^{k}\right\|_{L^{2}(\Omega)}\right)\left(\left\|\partial_{t} \theta^{k+\frac{1}{2}}+\partial_{t} \theta^{k-\frac{1}{2}}\right\|_{L^{2}(\Omega)}\right) \\
& \leq K \tau \sum_{k=1}^{n}\left[\left\|\frac{\partial^{2} \rho^{k}}{\partial t^{2}}\right\|_{L^{2}(\Omega)}^{2}+\left\|R_{1}^{k}\right\|_{L^{2}(\Omega)}^{2}+\left\|\partial_{t} \theta^{k+\frac{1}{2}}\right\|_{L^{2}(\Omega)}^{2}+\left\|\partial_{t} \theta^{k-\frac{1}{2}}\right\|_{L^{2}(\Omega)}^{2}\right] \\
& \leq K\left\{h_{\sigma}^{2 r+2}+\tau^{4}+\tau \sum_{k=1}^{n}\left[\left\|\partial_{t} \theta^{k+\frac{1}{2}}\right\|_{L^{2}(\Omega)}^{2}+\left\|\partial_{t} \theta^{k-\frac{1}{2}}\right\|_{L^{2}(\Omega)}^{2}\right]\right\}
\end{aligned}
$$


For $E_{3}$, we have the following estimate

$$
\begin{aligned}
E_{3}= & 2 \sum_{k=1}^{n}\left(\beta_{k} \nabla \cdot \rho^{k, \frac{1}{4}}, \nabla \cdot \theta^{k+\frac{1}{2}}-\nabla \cdot \theta^{k-\frac{1}{2}}\right) \\
= & \sum_{k=1}^{n}\left[\left(\beta_{k} \nabla \cdot \rho^{k+\frac{1}{2}}, \nabla \cdot \theta^{k+\frac{1}{2}}\right)-\left(\beta_{k-1} \nabla \cdot \rho^{k-\frac{1}{2}}, \nabla \cdot \theta^{k-\frac{1}{2}}\right)\right] \\
& -\tau \sum_{k=1}^{n}\left(\beta_{k-1} \nabla \cdot \frac{\rho^{k+1}+\rho^{k}-\rho^{k-1}-\rho^{k-2}}{\tau}, \nabla \cdot \theta^{k-\frac{1}{2}}\right) \\
= & \left(\beta_{n} \nabla \cdot \rho^{n+\frac{1}{2}}, \nabla \cdot \theta^{n+\frac{1}{2}}\right)-\left(\beta_{0} \nabla \cdot \rho^{\frac{1}{2}}, \nabla \cdot \theta^{\frac{1}{2}}\right) \\
& -\tau \sum_{k=1}^{n}\left(\beta_{k-1} \nabla \cdot \frac{\rho^{k+1}+\rho^{k}-\rho^{k-1}-\rho^{k-2}}{\tau}, \nabla \cdot \theta^{k-\frac{1}{2}}\right) \\
\leq & \varepsilon\left\|\nabla \cdot \theta^{n+\frac{1}{2}}\right\|_{L^{2}(\Omega)}^{2}+K\left\{\tau \sum_{k=1}^{n}\left\|\nabla \cdot \theta^{k-\frac{1}{2}}\right\|_{L^{2}(\Omega)}^{2}+h_{\sigma}^{2 r_{1}}\right\}
\end{aligned}
$$

where we have used Lemma 2.1 in the last inequality.

In addition, we know that

$$
\begin{aligned}
E_{4}= & \tau \sum_{k=1}^{n}\left[\left(\partial_{t}^{2}(\tilde{\mathcal{A}} \boldsymbol{\sigma})^{k}-\tilde{\mathcal{A}}_{k} \partial_{t}^{2} \boldsymbol{\sigma}^{k}, \partial_{t} \theta^{k+\frac{1}{2}}\right)-\left(\partial_{t}^{2}(\tilde{\mathcal{A}} \boldsymbol{\sigma})^{k-1}-\tilde{\mathcal{A}}_{k-1} \partial_{t}^{2} \boldsymbol{\sigma}^{k-1}, \partial_{t} \theta^{k-\frac{1}{2}}\right)\right] \\
& -\tau \sum_{k=1}^{n}\left(\left(\partial_{t}^{2}(\tilde{\mathcal{A}} \boldsymbol{\sigma})^{k}-\tilde{\mathcal{A}}_{k} \partial_{t}^{2} \boldsymbol{\sigma}^{k}\right)-\left(\partial_{t}^{2}(\tilde{\mathcal{A}} \boldsymbol{\sigma})^{k-1}-\tilde{\mathcal{A}}_{k-1} \partial_{t}^{2} \boldsymbol{\sigma}^{k-1}\right), \partial_{t} \theta^{k-\frac{1}{2}}\right) \\
\leq & \varepsilon\left\|\partial_{t} \theta^{n+\frac{1}{2}}\right\|_{L^{2}(\Omega)}^{2}+K \tau\left\{\sum_{k=1}^{n}\left\|\partial_{t} \theta^{k-\frac{1}{2}}\right\|_{L^{2}(\Omega)}^{2}+\tau^{4}\right\}
\end{aligned}
$$

and

$$
\begin{aligned}
E_{5}= & 2 \sum_{k=1}^{n}\left(\beta_{k} \nabla \cdot \boldsymbol{\sigma}^{k, \frac{1}{4}}-(\beta \nabla \cdot \boldsymbol{\sigma})^{k, \frac{1}{4}}, \nabla \cdot \theta^{k+\frac{1}{2}}-\nabla \cdot \theta^{k-\frac{1}{2}}\right) \\
= & 2 \sum_{k=1}^{n}\left[\left(\beta_{k} \nabla \cdot \boldsymbol{\sigma}^{k, \frac{1}{4}}-(\beta \nabla \cdot \boldsymbol{\sigma})^{k, \frac{1}{4}}, \nabla \cdot \theta^{k+\frac{1}{2}}\right)\right. \\
& \left.-\left(\beta_{k-1} \nabla \cdot \boldsymbol{\sigma}^{k-1, \frac{1}{4}}-(\beta \nabla \cdot \boldsymbol{\sigma})^{k-1, \frac{1}{4}}, \nabla \cdot \theta^{k-\frac{1}{2}}\right)\right] \\
& -2 \sum_{k=1}^{n}\left(\beta_{k} \nabla \cdot \boldsymbol{\sigma}^{k, \frac{1}{4}}-(\beta \nabla \cdot \boldsymbol{\sigma})^{k, \frac{1}{4}}-\left(\beta_{k-1} \nabla \cdot \boldsymbol{\sigma}^{k-1, \frac{1}{4}}\right.\right. \\
& \left.\left.-(\beta \nabla \cdot \boldsymbol{\sigma})^{k-1, \frac{1}{4}}\right), \nabla \cdot \theta^{k+\frac{1}{2}}\right) \\
\leq & \varepsilon\left\|\nabla \cdot \theta^{n+\frac{1}{2}}\right\|_{L^{2}(\Omega)}^{2}+K \tau\left\{\sum_{k=1}^{n}\left\|\nabla \cdot \theta^{k-\frac{1}{2}}\right\|_{L^{2}(\Omega)}^{2}+\tau^{4}\right\} .
\end{aligned}
$$


Therefore, substituting these estimates into (3.10), for sufficiently small $\varepsilon$, we can obtain the estimate

$$
\begin{aligned}
& \left\|\partial_{t} \theta^{n+\frac{1}{2}}\right\|_{L^{2}(\Omega)}^{2}+\left\|\nabla \cdot \theta^{n+\frac{1}{2}}\right\|_{L^{2}(\Omega)}^{2} \\
& \leq K\left\{\tau \sum _ { k = 1 } ^ { n } \left[\left\|\partial_{t} \theta^{k-\frac{1}{2}}\right\|_{L^{2}(\Omega)}^{2}+\left\|\partial_{t} \theta^{k+\frac{1}{2}}\right\|_{L^{2}(\Omega)}^{2}+\left\|\nabla \cdot \theta^{k-\frac{1}{2}}\right\|_{L^{2}(\Omega)}^{2}\right.\right. \\
& \left.\left.\quad+\left\|\nabla \cdot \theta^{k-\frac{1}{2}}\right\|_{L^{2}(\Omega)}^{2}\right]+h_{\sigma}^{2 r_{1}}+\tau^{4}\right\} .
\end{aligned}
$$

Using Gronwall's lemma, we get the estimate

$$
\left\|\partial_{t} \theta\right\|_{\tilde{L}^{\infty}\left(L^{2}(\Omega)\right)}+\|\nabla \cdot \theta\|_{\tilde{L}^{\infty}\left(L^{2}(\Omega)\right)} \leq K\left\{h_{\sigma}^{r_{1}}+\tau^{2}\right\} .
$$

Hence, by use of (2.9) and Lemma 3.1 again, we can obtain the estimate (3.8a).

Finally, we show that the estimate (3.8b) holds.

Set $\xi^{n}=u_{h}^{n}-P_{M} u^{n}$ and $\eta^{n}=u^{n}-P_{M} u^{n}$. Subtracting (3.2b) from (3.5b), using the definition of operator $P_{M}$ and (2.10a), we have

$$
\left(\partial_{t}^{2} \xi^{n}, v_{h}\right)=-\left(\beta_{n} \nabla \cdot\left(\sigma_{h}-\sigma\right)^{n, \frac{1}{4}}+R_{2}^{n}, v_{h}\right)-\left((\beta \nabla \cdot \sigma)^{n, \frac{1}{4}}-\beta_{n} \nabla \cdot \boldsymbol{\sigma}^{n, \frac{1}{4}}, v_{h}\right) .
$$

Taking $v_{h}=2 \delta_{t} \xi^{n}$ in (3.14), we have

$$
\begin{aligned}
&\left\|\partial_{t} \xi^{n+\frac{1}{2}}\right\|_{L^{2}(\Omega)}^{2}-\left\|\partial_{t} \xi^{n-\frac{1}{2}}\right\|_{L^{2}(\Omega)}^{2} \\
&=-\tau\left(\beta_{n} \nabla \cdot\left(\sigma_{h}-\sigma\right)^{n, \frac{1}{4}}+R_{2}^{n}, \partial_{t} \xi^{n+\frac{1}{2}}+\partial_{t} \xi^{n-\frac{1}{2}}\right) \\
& \quad-\tau\left((\beta \nabla \cdot \sigma)^{n, \frac{1}{4}}-\beta_{n} \nabla \cdot \boldsymbol{\sigma}^{n, \frac{1}{4}}, \partial_{t} \xi^{n+\frac{1}{2}}+\partial_{t} \xi^{n-\frac{1}{2}}\right) \\
& \leq K \tau\left\{\left\|\nabla \cdot\left(\sigma_{h}-\sigma\right)^{n, \frac{1}{4}}\right\|_{L^{2}(\Omega)}^{2}+\left\|(\beta \nabla \cdot \sigma)^{n, \frac{1}{4}}-\beta_{n} \nabla \cdot \sigma^{n, \frac{1}{4}}\right\|_{L^{2}(\Omega)}^{2}\right. \\
&\left.+\left\|R_{2}^{n}\right\|_{L^{2}(\Omega)}^{2}+\left\|\partial_{t} \xi^{n+\frac{1}{2}}\right\|_{L^{2}(\Omega)}^{2}+\left\|\partial_{t} \xi^{n-\frac{1}{2}}\right\|_{L^{2}(\Omega)}^{2}\right\}
\end{aligned}
$$

Summing from 1 to $n$, and using the estimate (3.8a), we can get

$$
\left\|\partial_{t} \xi^{n+\frac{1}{2}}\right\|_{L^{2}(\Omega)}^{2} \leq K\left\{\tau \sum_{l=1}^{n}\left[\left\|\partial_{t} \xi^{l+\frac{1}{2}}\right\|_{L^{2}(\Omega)}^{2}+\left\|\partial_{t} \xi^{l-\frac{1}{2}}\right\|_{L^{2}(\Omega)}^{2}\right]+h_{\sigma}^{2 r_{1}}+\tau^{4}\right\} .
$$

Using Gronwall's lemma and Lemma 3.1, we can obtain the estimate (3.8b). Hence we complete the proof of Theorem 3.2.

\section{NUMERICAL EXAMPLE}

In this section, we give some numerical results to confirm our theoretical analysis. Consider the following hyperbolic equation

$$
\begin{cases}\partial_{t t} u-\Delta u=f, & \text { in } \quad \Omega \times(0, T] \\ u(x, t)=0, & \text { on } \quad \partial \Omega, t>0 .\end{cases}
$$


TABLE I. $\quad T=1.0, h=\tau$.

\begin{tabular}{lccccc}
\hline & $h=\frac{1}{10}$ & $h=\frac{1}{20}$ & $h=\frac{1}{40}$ & $h=\frac{1}{80}$ & Rates \\
\hline$L^{\infty}\left(L^{\infty}\right)$ & $9.0785 \mathrm{e}-002$ & $2.4725 \mathrm{e}-002$ & $6.7232 \mathrm{e}-003$ & $3.2995 \mathrm{e}-003$ & - \\
$L^{\infty}\left(L^{2}\right)$ & $4.0631 \mathrm{e}-002$ & $1.0580 \mathrm{e}-002$ & $2.6749 \mathrm{e}-003$ & $7.6396 \mathrm{e}-004$ & 1.9110 \\
$\tilde{L}^{\infty}\left(L^{2}\right)$ & $2.3521 \mathrm{e}-001$ & $6.3949 \mathrm{e}-002$ & $1.6368 \mathrm{e}-002$ & $4.1383 \mathrm{e}-003$ & 1.9429 \\
$\star$ & $2.4321 \mathrm{e}-000$ & $1.3122 \mathrm{e}-000$ & $6.8146 \mathrm{e}-001$ & $3.4722 \mathrm{e}-001$ & 0.9361 \\
\hline
\end{tabular}

Using fully-discrete SPDME scheme, we compute the finite element approximate value $\sigma_{h}$ of the exact solution $\sigma=-\nabla u$.

In this experiment, $\Omega=[0,1] \times[0,1]$. The right-hand side, initial conditions of the system (4.1) are selected by the exact solution $u=e^{-t} \sin ^{2}(\pi x) \sin ^{2}(\pi y)$ and $\sigma=-\nabla u$. We choose the finite element space of piecewise linear polynomials. The following numerical results can be obtained (see Table 1) where $\star$ denote the error $\left\|\partial_{t}\left(\sigma-\sigma_{h}\right)\right\|_{\tilde{L}^{\infty}\left(L^{2}(\Omega)\right)}+\left\|\nabla \cdot\left(\sigma-\sigma_{h}\right)\right\|_{\tilde{L}_{\left(L^{2}(\Omega)\right)}}$.

From Table I, we can see that the convergence rates of $L^{\infty}\left(L^{2}\right)$-norm and $\tilde{L}^{\infty}\left(L^{2}\right)$-norm errors can nearly reach the optional order under the condition $h=\tau$. The numerical results denoted by " $\star$ " suggest that the error convergence order is approximate one, which is coincided with our theoretical analysis.

The authors would like to express their sincere thanks to the referees for their very helpful comments and suggestions, which greatly improved the quality of this paper.

\section{References}

1. T. Dupont, $L^{2}$-estimates for Galerkin methods for second order hyperbolic equations, SIAM J Numer Anal 10 (1973), 880-889.

2. G. A. Baker, Error estimate for finite element methods for second order hyperbolic equations, SIAM J Numer Anal 13 (1976), 564-576.

3. Y. R. Yuan, Stability and convergence of the finite element method for a class of second order nonlinear hyperbolic equations, Math Numer Sinica 5 (1983), 149-161.

4. Y. R. Yuan and H. Wang, The error estimate for the finite element method for nonlinear hyperbolic equations, J Sys Sci Math Sci 7 (1985), 161-171.

5. L. C. Cowsar, T. F. Dupont, and M. F. Wheeler, A priori estimates for mixed finite element methods for the wave equation, Comput Methods Appl Mech Eng 82 (1990), 205-222.

6. T. Geveci, On the application of mixed finite element methods to the wave equation, Math Model Numer Anal 22 (1988), 243-250.

7. Y. P. Chen and Y. Q. Huang, Mixed finite element method for nonlinear hyperbolic equations, Numer Math A: J Chin Univ (in Chinese) 22 (2000), 63-69.

8. D. P. Yang, A splitting positive definite mixed element method for miscible displacement of compressible flow in porous media, Numer Methods Partial Differential Equations 17 (2001), 229-249.

9. R. A. Adams, Sobolev spaces, Academic, New York, 1975.

10. J. L. Lions and E. Magenes, Nonhomogeneous boundary value problems and applications I, Springer Verlag, Berlin, 1972.

11. F. Brezzi, Douglas J. Jr., R. Duran, and M. Fortin, Mixed finite elements for sencond order elliptic problems in three variables, Numer Math 51 (1987), 237-250.

12. F. Breezi, Douglas J. Jr., M. Fortin, and L. D. Marini, Efficient rectangular mixed finite elements in two and three space variables, RAIRO Model Math Anal Numer 4 (1987), 581-604. 
13. F. Breezi, Douglas J. Jr., and L. D. Marini, Two families of mixed finite elements for second order elliptic problems, Numer Math 47 (1985), 217-235.

14. P. A. Raviart and J. M. Thomas, A mixed finite element method for 2nd order elliptic problems, Mathematical aspects of finite element methods, Lecture Notes in Math, Vol. 606, Springer Verlag, Berlin, 1977, pp. 292-315.

15. J. C. Nedelec, Mixed finite element, Numer Math 35 (1980), 315-341.

16. P. G. Ciarlet, The finite element methods for elliptic problems, North-Holland, New York, 1978. 